\title{
THE INFLUENCE OF A TRANSVERSE MAGNETIC FIELD ON
} THE CONDUCTIVITY OF THIN METALLIC FILMS

\author{
E. H. SONDHEIMER
}
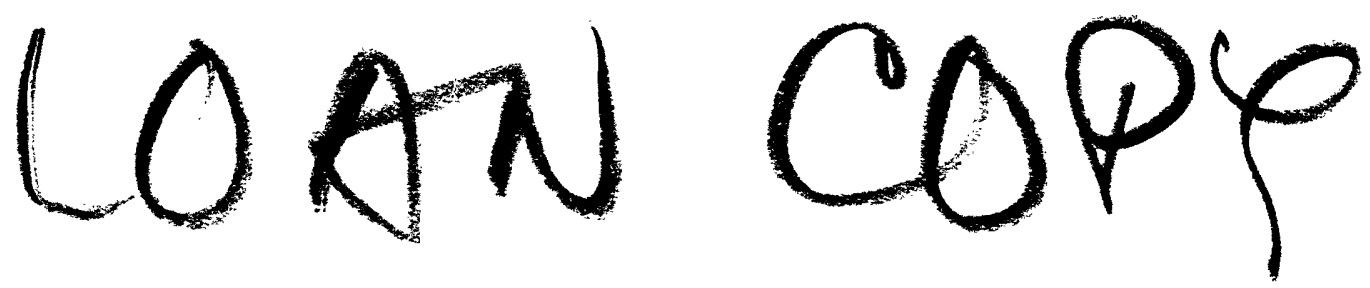

TECHNICAL REPORT NO. 161

MAY 31, 1950

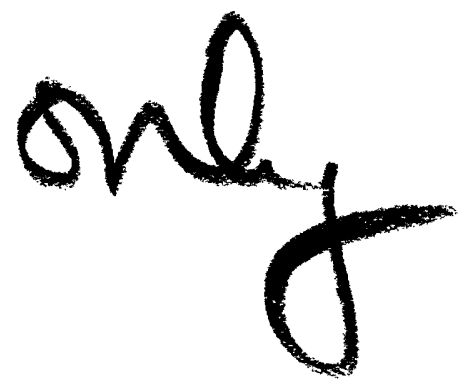

\footnotetext{
RESEARCH LABORATORY OF ELECTRONICS MASSACHUSETTS INSTITUTE OF TECHNOLOSY
} 
The research reported in this document was made possible through support extended the Massachusetts Institute of Technology, Research Laboratory of Electronics, jointly by the Army Signal Corps, the Navy Department (Office of Naval Research) and the Air Force (Air Materiel Command), under Signal Corps Contract No. W36-039-sc-32037, Project No. 102B; Department of the Army Project No. 3-99-10-022. 


\title{
MASSACHUSETTS INSTITUTE OF TECHNOLOGY
}

\section{RESEARCH LABORATORY OF ELECTRONICS}

Technical Report No. 161

May 31, 1950

THE INFLUENCE OF A TRANSVERSE MAGNETIC FIELD ON THE CONDUCTIVITY OF THIN METALLIC FILMS

E. H. Sondheimer

\begin{abstract}
The influence of a transverse magnetic field on the conduction properties of thin metallic films, of thickness comparable with the free path of the conduction electrons, is investigated. It is shown that, owing to scattering of electrons at the boundaries of the film, the Hall coefficient is increased, and the electrical resistance oscillates with the strength of the applied magnetic field.
\end{abstract}




\section{THE INFLUENCE OF A TRANSVERSE MAGNETIC FIELD ON THE CONDUCTIVITY OF THIN METALLIC FILMS}

\section{Introduction}

In the theory of metals it is usual to assume that the conduction electrons may be treated as if they were free, with the energy proportional to the square of the wavevector. This assumption is sufficient to explain most of the conduction phenomena, but it leads to a zero change of resistance in a magnetic field; and to explain the usual type of magneto-resistance effect ( 1 ) it is necessary to employ more complicated models which take into account the departure of the energy surfaces from spherical symmetry (2). However, in proving that there is no magneto-resistance effect for the free-electron model it is tacitly assumed that all the dimensions of the specimen considered are large compared with the free path of the conduction electrons. This requirement is fulfilled under ordinary conditions, but it may break down in the case of thin films or wires at very low temperatures; and under such conditions, where boundary scattering of electrons plays an essential part in determining the resistance, the alteration of the free electron trajectories in a magnetic field may lead to a finite magneto-resistance effect. This is a 'geometrical' effect which is of a totally different type from the ordinary increase in resistance observed in the bulk metal, and, since it is essentially classical in nature, it is much simpler to understand. The details of the phenomena observed in any particular case depend, of course, upon the shape of the specimen and upon the relative configurations of specimen, electric current and magnetic field.

An effect of this type was first observed by MacDonald (3) who found that the resistance of a thin sodium wire at low temperatures decreased when a longitudinal magnetic field was applied. In the present paper, we discuss only the case of a thin film placed in a magnetic field which is perpendicular to the plane of the film. Although this is perhaps not the simplest case to visualize, it is the easiest to analyze mathematically, and an exact solution may be obtained, assuming only that the conduction electrons are quasi-free and that a time of relaxation may be defined for their collisions with the ionic lattice of the metal. These assumptions are sufficient to bring out all the essential features of the phenomena; a more general model, which leads also to a finite magnetoresistance effect in the bulk metal, will be examined in a later paper.

The increase in resistance of a thin metallic film in the absence of a magnetic field has been discussed by Fuchs (4), and the present theory is a simple generalization of Fuchs' analysis. General formulas for the electrical conductivity and the Hall coefficient in a magnetic field of arbitrary magnitude are derived in Sect. 2, both for the case in which the electrons suffer diffuse reflection at the surface of the film and for the more general case of partially elastic surface scattering. In Sect. 3 we discuss the Hall coefficient in the limit of small magnetic fields, and we find that it shows an increase above the bulk value analogous to the increase of the zero-field electrical resistance. The field variation of the magnetic effects is considered in Sect. 4, and it is shown in 
particular that, for films whose thickness is small compared with the free path, the resistance oscillates with the strength of the applied magnetic field. By measuring the position of the resistance maxima and minima it is possible to obtain a direct estimate of the momentum of the electrons at the surface of the Fermi distribution, and hence of the number of conduction electrons per unit volume.

\section{General Formulas for the Magnetic Effects}

2.1. We consider a metal film of thickness a, with its surfaces parallel to the xyplane, which is subjected to an electric field $\left(\mathcal{E}_{x}, \mathcal{E}_{y}, 0\right)$ in the plane of the film and a transverse magnetic field $(0,0, \mathrm{H})$. (For this particular arrangement, the condition curl $\underset{\sim}{\mathbb{E}}=0$ ensures that the electric field components are constant across the thickness of the film.) The conduction electrons in the metal are regarded as free, in the sense that the energy $\mathrm{E}$ is related to the wave-vector $\underset{\sim}{\mathrm{k}}$ by $\mathrm{E}=\mathrm{h}^{2}|\underset{\sim}{\mathrm{k}}|^{2} /\left(8 \pi^{2} \mathrm{~m}\right)$, where $\mathrm{m}$ is the effective mass of an electron. The distribution function $f$ of the electrons is written in the form

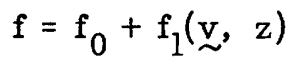

where $f_{0}$ is the Fermi function $l /\left(e^{(E-\zeta) / k T}+1\right)(\zeta$ is the Fermi energy level), $\underset{\sim}{v}$ is the velocity of an electron, and $f_{1}$ is a function of $\underset{\sim}{v}$ and $z$ which has to be determined. $f_{0}$ depends on the absolute value of $\underset{\sim}{\mathrm{v}}$ only.

Under the combined action of the applied fields and the collisions of the electrons with the lattice a steady state is set up, and the distribution function in the steady state is determined by the Boltzmann equation (5)

$$
-\frac{2 \pi \epsilon}{\mathrm{h}}\left(\underset{\sim}{\mathcal{E}}+\frac{\mathrm{l}}{\mathrm{c}} \mathrm{v} \times \underset{\sim}{\mathrm{H}}\right) \cdot \operatorname{grad}_{\underset{\sim}{\mathrm{k}}} \mathrm{f}+\underset{\sim}{\mathrm{v}} \cdot \operatorname{grad}_{\underset{\mathrm{r}}{\mathrm{f}} \mathrm{f}=-\frac{\mathrm{f}-\mathrm{f}_{0}}{\tau}}
$$

where $-\epsilon$ is the electronic charge and $\tau$ is the time of relaxation of the conduction electrons. $f$ is regarded as a function of the wave-vector $\underset{\sim}{\mathrm{k}}$ and the space-vector $\underset{\sim}{\mathrm{r}}$, and $\mathrm{T}$ is assumed to depend on the absolute value of $\underset{\sim}{\mathrm{k}}$ only. The term $\underset{\sim}{\mathrm{v}} \cdot \operatorname{grad} \underset{\underset{r}{\mathbf{r}}}{\mathrm{f}}$ has to be included to take account of the nonuniform space distribution of the electrons in the z-direction.

On combining Eqs. $\mathrm{l}$ and 2 , using the relation $\mathrm{hk} \underset{\sim}{ }=2 \pi \mathrm{mv}$ which holds for free electrons, and neglecting as usual the product of $\mathcal{E}_{\text {with }} f_{1}$ but retaining all the terms involving $H, f_{1}$ is found to satisfy the equation

$$
\frac{\partial f_{1}}{\partial z}+\frac{f_{1}}{\tau v_{z}}-\frac{\epsilon H}{m c v_{z}}\left(v_{y} \frac{\partial f_{1}}{\partial v_{x}}-v_{x} \frac{\partial f_{1}}{\partial v_{y}}\right)=\frac{\epsilon}{m v_{z}}\left(\varepsilon_{x} \frac{\partial f_{0}}{\partial v_{x}}+\varepsilon_{y} \frac{\partial f_{0}}{\partial v_{y}}\right) \text {. }
$$

2.2. To solve this equation we put

$$
f_{1}=\left(v_{x} c_{1}+v_{y} c_{2}\right) \frac{\partial f_{0}}{\partial v}
$$


where $\mathrm{v}$ is the absolute value of $\underset{\sim}{\mathrm{v}}$, and where $\mathrm{c}_{1}$ and $\mathrm{c}_{2}$ are functions of $\mathrm{v}, \mathrm{v}_{\mathrm{z}}$ and $\mathrm{z}$ which do not depend explicitly on $\mathrm{v}_{\mathrm{x}}$ and $\mathrm{v}_{\mathrm{y}}$. From Eqs. 3 and 4 we obtain the following simultaneous equations for $c_{1}$ and $c_{2}$ :

$$
\begin{aligned}
& \frac{\partial c_{1}}{\partial z}+\frac{c_{1}}{\tau v_{z}}+\frac{\epsilon H}{m c v_{z}} c_{2}=\frac{\epsilon}{m v_{z}} \mathcal{E}_{x} \\
& \frac{\partial c_{2}}{\partial z}+\frac{c_{2}}{\tau v_{z}}-\frac{\epsilon H}{m c v_{z}} c_{1}=\frac{\epsilon}{m v_{z}} \varepsilon_{y} .
\end{aligned}
$$

Introducing the complex quantities $g=c_{1}-i c_{2}, \mathcal{F}=\mathcal{E}_{\mathrm{x}}-i \mathcal{E}_{\mathrm{y}}$, we may write these equations in the compact form

$$
\frac{\partial g}{\partial z}+\frac{g}{\tau v_{z}}+\frac{i \epsilon H}{m c v_{z}} g=\frac{\epsilon}{m v_{z}} f
$$

The general solution is

$$
g=\frac{\epsilon \tau \mathcal{F}}{m v\left(1+\frac{i \epsilon H \tau}{m c}\right)}\left[1+F(\underset{\sim}{v}) \exp \left(-\left(1+\frac{i \in H \tau}{m c}\right) \frac{z}{\tau v_{z}}\right)\right]
$$

where $\mathrm{F}$ is an arbitrary function of $\mathrm{v}$ and $\mathrm{v}_{\mathrm{z}}$.

The boundary conditions which are used to determine $F$ depend on the nature of the scattering at the surface of the film. If we assume, as we shall do for the present, that the electrons are scattered entirely at random, with complete loss of their drift velocity, the distribution function of the electrons leaving each surface of the film must be independent of their direction of motion. This requirement is obviously satisfied if we take

$$
F(\underset{\sim}{v})=-1 \quad \text { for all } \underset{\sim}{v} \text { such that } v_{z}>0
$$

and

$$
F(\underset{\sim}{v})=-\exp \left(\left(1+\frac{i \epsilon H \tau}{m c}\right) \frac{a}{\tau v_{z}}\right) \quad \text { for all } \underset{\sim}{v} \text { such that } v_{z}<0
$$

since these equations ensure that $g$ (and therefore $f_{1}$ ) vanishes for electrons leaving the surfaces $z=0$ and $z=a$ respectively.

2.3. The current density $\left(\mathrm{J}_{\mathrm{x}}, \mathrm{J}_{\mathrm{y}}, 0\right)$ can now be calculated. For example, we have

$$
J_{x}=-2 \epsilon\left(\frac{m}{h}\right)^{3} \iiint v_{x} f d v_{x} d v_{y} d v_{z} \text {. }
$$

Using Eqs. 1 and 4, introducing polar coordinates $(v, \theta, \widetilde{\omega})$ in the $\underset{\sim}{v}$-space (with $\left.\mathrm{v}_{\mathrm{z}}=\mathrm{v} \cos \theta\right)$ and integrating over $\tilde{\omega}$, this becomes

$$
\mathrm{J}_{\mathrm{x}}=-2 \pi \epsilon\left(\frac{\mathrm{m}}{\mathrm{h}}\right)^{3} \iint \mathrm{v}^{4} \sin ^{3} \theta \mathrm{c}_{1} \frac{\partial \mathrm{f}_{0}}{\partial \mathrm{v}} \mathrm{dv} \mathrm{d} \theta .
$$


There is a corresponding expression for $J_{y}$ with $c_{1}$ replaced by $c_{2}$.

Introducing the complex current $f=\mathrm{J}_{\mathrm{x}}-i \mathrm{~J}_{\mathrm{y}}$, and integrating over $\mathrm{v}$ by means of the formula

$$
-\int \psi(v) \frac{\partial f_{0}}{\partial v} d v=\psi(\bar{v})
$$

which holds for a degenerate electron gas, $\bar{v}$ being the velocity at the surface of the Fermi distribution, we obtain

$$
g=2 \pi \epsilon\left(\frac{\mathrm{m}}{\mathrm{h}}\right)^{3} \overline{\mathrm{v}}^{4} \int_{0}^{\pi} \sin ^{3} \theta \mathrm{gd} \theta .
$$

For comparison with experiment we require the mean current $\bar{g}$, averaged across the thickness of the film. Combining Eqs. 8, 9, 10 and 11, we obtain after some calculation

$$
\bar{g}=\frac{1}{a} \int_{0}^{a} g \mathrm{dz}=\frac{\mathrm{K} \sigma_{0}^{\mathcal{F}}}{\phi(s)}
$$

where

$$
\frac{1}{\phi(s)}=\frac{1}{s}-\frac{3}{8 s^{2}}+\frac{3}{2 s^{2}} \int_{1}^{\infty}\left(\frac{1}{t^{3}}-\frac{1}{t^{5}}\right) e^{-s t} d t
$$

and where $\mathrm{s}$ is a complex variable defined by

$$
s=K+i \beta
$$

with

$$
\mathrm{K}=\frac{\mathrm{a}}{\mathrm{l}}, \quad \beta=\frac{\mathrm{a}}{\mathrm{r}},
$$

$\ell=\tau \bar{v}$ being the free path of the conduction electrons, and $r=m \bar{v} c / \epsilon H$ being the radius of the circular orbit of an electron in a magnetic field $\mathrm{H}$. We have also used the expressions

$$
\sigma_{0}=\frac{n \epsilon^{2} l}{m \bar{v}}, \quad n=\frac{8 \pi}{3}\left(\frac{m \bar{v}}{h}\right)^{3}
$$

for the conductivity of the bulk metal and for the number of electrons per unit volume.

The formal similarity between Eq. 12 of the present paper and Eq. 17 of Fuchs' paper (4) should be noted.

2.4. Equation 12 contains all the results required for comparison with experiment. The electrical conductivity $\sigma$, for example, is measured by applying an electric field in say the $\mathrm{x}$-direction and observing the current in this direction, no electric current being allowed to flow in the transverse direction. We therefore have $\sigma=\mathbb{R}(\bar{g}) / R(\mathcal{F})$, with $\mathscr{Q}(\bar{g})=0$, where $\mathcal{R}$ and $\mathcal{Q}$ denote the real and imaginary parts respectively. Using 
the expression (12) for $\bar{g}$ and eliminating $\mathcal{F}$, we obtain for the ratio of the resistivity $1 / \sigma$ to that of the bulk metal $1 / \sigma_{n}$

$$
\frac{\sigma_{0}}{\sigma}=\frac{R\{\phi(s)\}}{\bar{K}}
$$

The Hall coefficient is defined by $\mathrm{A}_{\mathrm{H}}=\mathcal{E}_{\mathrm{y}} / \mathrm{H} \mathrm{J}_{\mathrm{x}}=-\mathcal{J}(\mathcal{F}) /\{\mathrm{H} R(\bar{g})\}$, where $\mathcal{E}_{\mathrm{y}}$ is the transverse electric field set up under the above experimental conditions, and we easily obtain

$$
\frac{\mathrm{A}_{\mathrm{H}}}{\mathrm{A}_{\mathrm{H}, 0}}=\frac{Q\{\phi(\mathrm{s})\}}{\beta}
$$

where $\beta$ is defined by (15), and where $A_{H, 0}=-1 /(n \in c)$ is the Hall coefficient of the bulk metal. The ratios $\sigma_{0} / \sigma$ and $\mathrm{A}_{\mathrm{H}} / \mathrm{A}_{\mathrm{H}, 0}$ are therefore functions of the two dimensionless parameters $K$ and $\beta$ only. Note that $K$ and $\beta$ are both proportional to the thickness of the film; also that $K$ depends upon the temperature (its temperature variation being the same as that of the bulk electrical resistance), whereas $\beta$ is proportional to the strength of the magnetic field but is independent of the temperature.

2.5. The theory is easily extended to apply to the more general case of partially elastic surface scattering, where a fraction $p$ of the electrons arriving at the surface of the metal is supposed to be specularly reflected, retaining its drift velocity, while the rest are scattered diffusely. The analysis is formally identical with that given by Fuchs (4) for the case of zero magnetic field and will not be presented here in detail. (Note that our notation differs somewhat from that used by Fuchs, a, $\ell$ and $p$ being used instead of $t, \lambda_{0}$ and $\epsilon_{.}$) It is found that all the results of the preceding sections apply, except that the function $\phi(s)$ defined by Eq. 13 has to be replaced by $\phi_{p}(s)$, where

$$
\frac{1}{\phi_{p}(s)}=\frac{1}{s}-\frac{3}{2 s^{2}}(1-p) \int_{1}^{\infty}\left(\frac{1}{t^{3}}-\frac{1}{t^{5}}\right) \frac{1-e^{-s t}}{1-p e^{-s t}} d t .
$$

This reduces to $\mathrm{Eq} .13$ when $\mathrm{p}=0$, and to the bulk metal value $1 / \mathrm{s}$ when $\mathrm{p}=1$.

\section{The Hall Coefficient in Small Magnetic Fields}

When $\beta=0, E q .17$ combined with Eqs. 13 or 19 leads at once to expressions for the conductivity of a thin film in the absence of a magnetic field which have already been given by Fuchs (Eqs. 18 and 22 of his paper). By expanding Eqs. 13 and 19 in ascending powers of $\beta$ and retaining only the linear term, corresponding expressions may be obtained for the Hall coefficient in the limit of vanishingly small magnetic fields. In this limit $A_{H} / A_{H, 0}$ is independent of $\beta$, and the resulting expression (for arbitrary $p$ ) may be written in the form 


$$
\frac{A_{H}}{A_{H, 0}}=\frac{1-\frac{3}{4 K}(1-p)-\frac{3}{2 K}(1-p)^{2} \sum_{m=1}^{\infty} p^{m-1}\left\{\frac{m^{4} K^{4}}{12} E i(-m K)-e^{-m K}\left(\frac{1}{2}-\frac{m K}{6}+\frac{m^{2} K^{2}}{12}-\frac{m^{3} K^{3}}{12}\right)\right\}}{\left[1-\frac{3}{8 K}(1-p)-\frac{3}{4 K}(1-p)^{2} \sum_{m=1}^{\infty} p^{m-1}\left\{E i(-m K)\left(m^{2} K^{2}-\frac{m^{4} K^{4}}{12}\right)-e^{-m K(}\left(\frac{1}{2}-\frac{5}{6} m K-\frac{m^{2} K^{2}}{12}+\frac{m^{3} K^{3}}{12}\right)\right\}\right]^{2}}
$$

where

$$
-\operatorname{Ei}(-u)=\int_{1}^{\infty} \frac{e^{-u t}}{t} d t
$$

When $\mathrm{p}=0, \mathrm{Eq} \cdot 20$ reduces to

$$
\frac{A_{H}}{A_{H, 0}}=\frac{1-\frac{1}{8} K^{3} E i(-K)-\frac{3}{4 K}\left(1-e^{-K}\right)-e^{-K\left(\frac{1}{4}-\frac{K}{8}+\frac{K^{2}}{8}\right)}}{\left[1-\frac{3}{4} \operatorname{Ei}(-K)\left(K-\frac{K^{3}}{12}\right)-\frac{3}{8 K}\left(1-e^{-K}\right)-e^{-K(}\left(\frac{5}{8}+\frac{K}{16}-\frac{K^{2}}{16}\right)\right]^{2}} .
$$

Equations 20 and 22 are to be compared with Eqs. 22 and 18 of Fuchs' paper. Figure 1 shows $A_{H} / A_{H, 0}$ as a function of $K$ for three values of $p$, and should be compared with Fig. 1 of Fuchs' paper. It is seen that the Hall coefficient of thin films shows an increase above the bulk value analogous to, but smaller than, the increase of the elec-

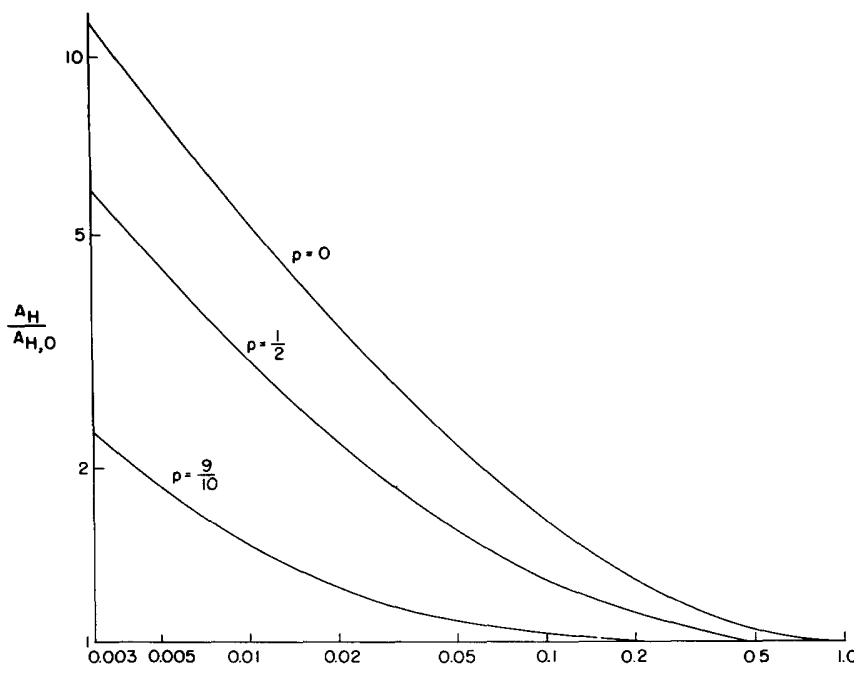

Fig. 1 The Hall coefficient of thin metallic films in the limit of small magnetic fields. trical resistivity*. For very small values of $\mathrm{K}, \mathrm{Eq} \cdot 20$ reduces to

$\frac{\mathrm{A}_{\mathrm{H}}}{\mathrm{A}_{\mathrm{H}, 0}}=\frac{4}{3} \frac{1-\mathrm{p}}{1+\mathrm{p}} \frac{1}{\mathrm{~K}\left(\log \frac{1}{\mathrm{~K}}\right)^{2}}$;

the corresponding expression for the electrical resistance is **

$\frac{\sigma_{0}}{\sigma}=\frac{4}{3} \frac{1-p}{1+p} \frac{1}{K \log \frac{1}{K}}=\left(\log \frac{1}{\bar{K}}\right) \frac{A_{H}}{A_{H, 0}}$.

To my knowledge there are at present no experimental results with which to compare the theoretical predictions outlined above.

* As $K$ decreases from large values, $A_{H}$ at first actually decreases slightly below the bulk value before increasing in the expected manner; the effect is very small, and is of no practical importance.

**The expression given by Fuchs for $\sigma_{0} / \sigma$ in the limit of small $\mathrm{K}$ (Eq. 23 of his paper) differs from Eq. 24 and is incorrect. 


\section{The Field Variation of the Conduction Phenomena}

4.1. The field dependence of $\sigma$ and $A_{H}$ is obtained from Eqs. 13, 17, 18 and 19 by keeping $\mathrm{K}$ constant and varying $\beta$. Various methods have been used to evaluate the theoretical expressions numerically. Considering for the present the case $p=0$ only, we find that, for small $|s|$ (that is, for $K^{2}+\beta^{2} \ll 1$ ), $\phi(s)$ is most conveniently evaluated by means of the power series

$$
\frac{1}{\phi(s)}=\frac{3}{4}(1-\gamma-\log s)+\frac{1}{2} s-\frac{1}{192} s^{2}(31-12 \gamma-12 \log s)+3 \sum_{n=3}^{\infty}(-1)^{n} \frac{s^{n}}{(n-2) n(n+2) !}
$$

where $\gamma$ is Euler's constant. For sufficiently large $|s|$, the asymptotic series

$$
\frac{1}{\phi(s)}=\frac{1}{s}-\frac{3}{8 s^{2}}+\frac{1}{16} e^{-s} \sum_{n=4}^{\infty}(-1)^{n} \frac{(n+4)(n-3)(n-1) !}{s^{n}}
$$

may be used. For intermediate values of $|s|$, neither of these series is of practical utility, and numerical integration is necessary. The integrals required are of the form

$$
\int_{1}^{\infty}\left(\frac{1}{t^{3}}-\frac{1}{t^{5}}\right) e^{-K t} \cos \beta t d t \text { and } \int_{1}^{\infty}\left(\frac{1}{t^{3}}-\frac{1}{t^{5}}\right) e^{-K t} \sin \beta t d t
$$

and cannot, in general, be expressed in terms of tabulated integrals. For small values of $K$, approximate values may be obtained by replacing $e^{-K t}$ by $1-K t+\frac{1}{2} K^{2} t^{2}$ and expressing the integrals in terms of the sine and cosine integrals for the argument $\beta$.

For non-zero values of $\mathrm{p}$ the calculations become very laborious. It is possible to write down expansions corresponding to Eqs. 25 and 26, but in general it is simplest to evaluate the real and imaginary parts of Eq. 19 by direct numerical integration.

4.2. The results of the calculations are shown in Figs. 2 to 5 for some typical values of the parameters, sufficient to illustrate the general behavior and to show the effect of varying $K$ and $p$. The curves of Fig. 2 show $\sigma_{0} / \sigma$ as a function of $\beta$ for $p=0$ and for three values of $K$, while those of Fig. 3 show $\sigma_{0} / \sigma$ as a function of $\beta$ for $K=0.02$ and for three values of $p$. Figures 4 and 5 give the corresponding curves for $A_{H} / A_{H, 0}$.

The most interesting result of these calculations is that the resistance of a thin metallic film oscillates with the strength of an applied transverse magnetic field. In the absence of a magnetic field the resistance is given by Fuchs' value; as the magnetic field is increased, the resistance increases initially and reaches its first maximum for a magnetic field such that $\beta$ is approximately unity. The higher oscillations are of roughly constant spacing (successive maxima occurring for $\beta=1,7,13,19, \ldots$ approximately) but decrease rapidly in amplitude, and in very strong magnetic fields the resistance tends to the constant asymptotic value

$$
\left(\frac{\sigma_{0}}{\sigma}\right)_{\beta=\infty}=1+\frac{3}{8 R}(1-p) \text {. }
$$



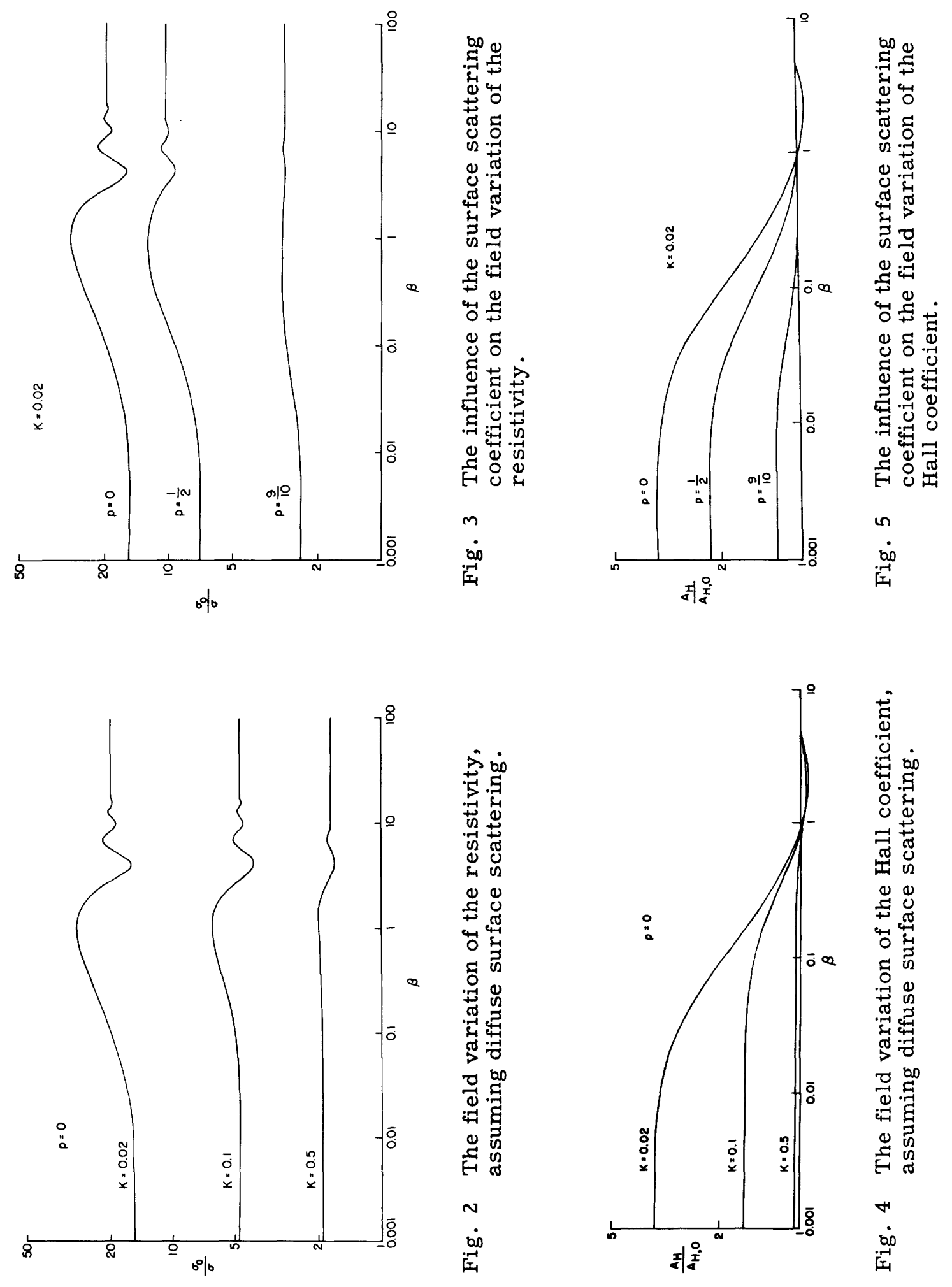
The oscillations die out and the resistance approaches the bulk value as $K$ tends to infinity or as $\mathrm{p}$ tends to unity; the position of the maxima and minima is not appreciably affected.

The Hall coefficient, on the other hand, does not oscillate, and, as $\beta$ increases from zero to infinity, $A_{H}$ decreases steadily from the value of Eqs. 20 or 22 to the bulk metal value *.

4.3. It is interesting to compare Eq. 24 for the conductivity of a thin film when $\mathrm{H}=0$ with $\mathrm{Eq} .27$ for the conductivity when $\mathrm{H}=\infty$. The logarithmic dependence of $\sigma$ on the ratio $\ell / a$ when $\ell \gg a$ is due to the fact that, in the absence of a magnetic field, the dominant contribution to the current arises from electrons which, after reflection at the surface of the film, move in directions so nearly parallel to the surface that they do not travel freely to the opposite surface but make their next collision in the bulk of the metal. Equation 27 shows that the contribution of these ' anomalous' electrons is eliminated in a strong transverse magnetic field, and the conductivity is given by an expression of the ordinary type, Eq. 16, with a length of the order of the thickness of the film playing the part of an effective free path of the conduction electrons.

The oscillations in conductivity which occur for intermediate values of the magnetic field are connected with the oscillations in speed of an electron moving in perpendicular electric and magnetic fields, as pointed out by Chambers (6). If we put $\varepsilon_{\mathrm{y}}=0$ and regard $\mathcal{E}_{X}$ as the resultant of the applied electric field and the Hall field, kinetic arguments may be used to show that the distribution function is given by (compare Eq. 28 of Chambers' paper)

$$
f(\underset{\sim}{v}, z)=f_{0}+\frac{\epsilon v_{0} \mathcal{E}_{x}}{m v v_{z}} \frac{\partial f_{0}}{\partial v} \int_{-z}^{0} \sin \left(\frac{\xi}{r \cos \theta}+\delta\right) e^{\frac{\xi}{\ell \cos \theta}} d \xi
$$

where $\mathrm{v}_{\mathrm{x}}=\mathrm{v}_{\mathrm{o}} \sin \delta, \mathrm{v}_{\mathrm{y}}=-\mathrm{v}_{\mathrm{o}} \cos \delta$, and the remaining symbols have their usual meaning. This expression is easily shown to be equivalent to the expression for $f$ obtained by combining Eqs. 1, 4, 8 and 9 of the present paper and putting $\mathcal{E}_{\mathrm{y}}=0$. The integrand of Eq. 28 represents the contribution to $f$ of electrons which have traveled freely to the point considered from a distance $\xi$ away in the z-direction, the limit of integration $-z$ corresponding to electrons which have traveled from the surface of the metal. The fluctuations in $f$ as a function of $z / r$ are due to the presence of an oscillating integrand in Eq. 28, and to the presence of the metal surface which provides an upper limit to the distance from which electrons can come to contribute to the current at $\mathrm{z}$. In a metal of infinite extent the limit of integration in Eq. 28 may be replaced by $-\infty$, and $f$ is then independent of $z$ and no longer fluctuates.

\footnotetext{
* More precisely, $A_{H}$ decreases to a value slightly below the bulk value, passes through a minimum and finally increases towards the bulk value. This behavior is similar to that of $A_{H}$ for $H=0$ as a function of $K$.
} 
4.4 Observations on the resistance oscillations predicted by the present theory may be used to estimate the momentum $m \bar{v}$ of the electrons at the surface of the Fermi distribution and hence the number $\mathrm{n}$ of conduction electrons per unit volume, which is related to $\mathrm{m} \overline{\mathrm{v}}$ by Eq. 16 . It should be noted that this estimate may be made without any accurate knowledge of the values of $K$ or $p$, since the values of $\beta$ for which the maxima and minima occur are almost independent of the values of the se parameters. It must, however, be borne in mind that a correction will have to be applied for the ordinary bulk magneto-resistance effect which is superimposed on the effect discussed above, and which will not be negligible at the low temperatures and high fields required to observe the oscillations even in the case of metals like the alkali metals for which it is most likely to be small (7). To estimate this correction theoretically it would be necessary to employ a more general model than the free-electron model used in the present theory, since this leads to a zero bulk effect. It is hoped to return to this question in a later paper.

The values of $\mathrm{n}$ obtained directly as explained above may be used to check the validity of the free-electron theory by comparing them with the values of $n$ obtained by combining estimates of $\ell$ obtained from the increase in resistance of a thin film in the absence of a magnetic field (assuming some value for $\mathrm{p}$ ) with estimates of $n^{2 / 3} \ell$ obtainable according to Eq. 16 from the bulk conductivity $\sigma_{0}$. Alternatively, the direct estimates of $\mathrm{n}$ may be used together with values of $\sigma_{0}$ to obtain $\ell$, and hence to obtain an estimate of the surface reflection coefficient $p$ from the observed increase in resistance in the fieldfree case (which depends sensitively on the value of $p$ ) (reference 4).

Finally, we give an estimate of the orders of magnitude which may occur in an actual experiment; these indicate that the resistance oscillations should be observable, though the experiment would probably not be easy. Note that the film thickness must be chosen to be neither too large, since it must be made small compared with the free path of the electrons, nor must it be too small, since otherwise it would not be possible to obtain sufficiently large values of $\beta$ with the magnetic fields that are obtainable in practice. If we choose $a=10^{-4} \mathrm{~cm}$, then a sufficiently large free path may be obtained by employing very pure unstrained specimens and liquid helium temperatures, Also, if the metal used is sodium, we have $n=2.5 \times 10^{22}$, and we estimate that $\beta=1$ in a magnetic field of 60,000 gauss. Smaller magnetic fields would be required in the case of metals with a smaller number of conduction electrons.

\section{Acknowledgment}

The work described in this paper was begun at the H. H. Wills Physical Laboratory, University of Bristol, England and was completed at the Research Laboratory of Electronics of the Massachusetts Institute of Technology, and the author would like to express his thanks to Professors N. F. Mott, J. C. Slater and A. G. Hill for the hospitality extended to him at their laboratories. He also wishes to thank Mr. R. G. Chambers for showing him the manuscript of his paper before publication, and Miss Elizabeth $\mathrm{J}$. 
Campbell of the Joint Computing Group of the Massachusetts Institute of Technology for patiently performing most of the heavy numerical work.

\section{References}

(1) P. Kapitza: Proc. Roy. Soc. Al23, 292 (1929).

(2) R. Peierls: Ann. d. Physik (5), 10, 97 (1931).

(3) D. K. C. MacDonald: Nature 163, 637 (1949).

(4) K. Fuchs: Proc. Camb. Phil. Soc. 34, 100 (1938).

(5) A. H. Wilson: The Theory of Metals, p. 158 (Cambridge University Press, London, 1936).

(6) R. G. Chambers, in course of publication.

(7) D. K. C. MacDonald: Proc. Phys. Soc. London A 63, 290 (1950). 
